Bicocca-FT-99-31

hep-ph/9910343

October 1999

\title{
The resummed thrust distribution in DIS
}

\author{
V. Antonelli用, M. Dasgupta and G. P. Salam \\ Dipartimento di Fisica, Università di Milano-Bicocca \\ and INFN, Sezione di Milano, Italy
}

\begin{abstract}
We present preliminary results on the resummation of leading and nextto-leading logarithms for the thrust distribution in deep inelastic scattering. Our predictions, expanded to $\mathcal{O}\left(\alpha_{\mathrm{S}}^{2}\right)$, are compared to corresponding results from the Monte Carlo programs DISASTER ++ and DISENT.

PACS numbers: 12.38.Bx, 12.38.Cy
\end{abstract}

\section{Introduction}

For some time now it has been standard practice in $e^{+} e^{-}$reactions to compare eventshape distributions with resummed perturbative predictions (see for instance [1], 2]). The resummation is necessary because in the two-jet limit (small values of the shape variable) the presence of large soft and collinear logarithms spoils the convergence of the fixed-order calculations. Such resummed analyses have led to valuable information about the strong coupling constant and also about non-perturbative effects.

At HERA, studies of event-shape distributions are being carried out by both collaborations [3] but as yet no perturbative resummed calculations exist for comparison. Here we present preliminary results on such a calculation [4].

In DIS, event shapes are defined in the current hemisphere of the Breit frame [5] to reduce contamination from remnant fragmentation which is beyond perturbative control. The distribution of emissions in the current hemisphere $\left(\mathcal{H}_{\mathrm{C}}\right)$ is analogous to that in a single hemisphere of $e^{+} e^{-}$. Differences arise from $e^{+} e^{-}$however because the momentum of the current quark (the quark struck by the photon) depends on remnant-hemisphere $\left(\mathcal{H}_{\mathrm{R}}\right)$ emissions through recoil effects. This necessitates a resummed treatment of the space-like branching of the incoming parton. Currently such a simultaneous resummation of the space-like and time-like (double logarithmic) contributions exists only for jet multiplicities [6], or for cross sections at large $x$ [7].

$\ddagger$ Talk presented by V. Antonelli at the UK Phenomenology Workshop on Collider Physics, September 1999, St. John's College, Durham. 
For the thrust $T$ (as defined in the following section) the recoil from remnant hemisphere emissions can be divided into two parts: a piece from soft and collinear emissions, which gives double logarithms $\alpha_{\mathrm{S}}^{n} \ln ^{2 n}(1-T)$, identical to those from half a hemisphere of $e^{+} e^{-}$, and a piece from purely collinear emissions which gives single logarithms $\alpha_{\mathrm{S}}^{n} \ln ^{n}(1-T)$ that can be identified with a change of scale in the parton distribution. This is outlined in the next section.

A valuable cross-check of any resummation is to expand it to NLO and compare the result to that from fixed order Monte Carlo programs such as DISASTER++ [8] and DISENT [9]. This is illustrated in section 3.

Finally in section 4 , we comment on possible future developments.

\section{The Thrust in DIS}

There are several possible definitions of the thrust in DIS, which differ according to a choice of axis and the normalisation. We here consider the thrust with respect to the photon axis, $\vec{n}_{\gamma}$, and normalised to $Q / 2$,

$$
T=\frac{2}{Q} \sum_{i \in \mathcal{H}_{\mathrm{C}}} \overrightarrow{k_{i}} \cdot \vec{n}_{\gamma},
$$

where the sum extends over all particles in the current hemisphere, $H_{C}$. At lowest order $T=1$, so the region requiring resummation will be that of $1-T$ close to zero.

Expressing all momenta in terms of their Sudakov components

$$
k_{i}=\alpha_{i} P+\beta_{i} P^{\prime}+k_{t, i}, \quad \alpha_{i} \beta_{i}=\vec{k}_{t, i}^{2} / Q^{2},
$$

where $P=\frac{1}{2} Q(1,0,0,-1)$ and $P^{\prime}=\frac{1}{2} Q(1,0,0,1)$ are along the remnant and current directions respectively, we can write the thrust as [10]

$$
1-T=\sum_{i \in \mathcal{H}_{\mathrm{R}}} \beta_{i}+\sum_{i \in \mathcal{H}_{\mathrm{C}}} \alpha_{i}+\alpha_{q}
$$

where the sums now run over emitted particles only and $\alpha_{q}$ is the $\alpha$ component of the current quark[s]. Dependence on the remnant hemisphere emissions arises both through the $\sum_{i \in \mathcal{H}_{\mathrm{R}}} \beta_{i}$ term and through

$$
\alpha_{q}=\frac{k_{t, q}^{2}}{\beta_{q}} \simeq\left|-\sum_{i} \vec{k}_{t, i}\right|^{2},
$$

(we have made use of the fact that $\beta_{q} \simeq 1$ ).

We now consider the cross section $\sigma(\tau)$ for $1-T$ to be smaller than $\tau$. Roughly it will contain virtual corrections from the exclusion of all emissions whose contribution to (3) is larger than $\tau$.

$\S$ The expression must be modified when the current quark goes into the remnant hemisphere — but such a situation is not relevant for small $1-T$. 
In the remnant hemisphere we exclude soft and collinear emissions, $k_{t}>\beta>\tau$, where the $k_{t}>\beta$ condition ensures that particle be in $\mathcal{H}_{\mathrm{R}}$. The integration over $k_{t}$ and $\beta$ gives a double logarithm. In $\mathcal{H}_{\mathrm{C}}$ we exclude the soft and collinear region $k_{t}>\alpha>\tau$. Again this gives us a double logarithm. The exclusion $\alpha_{q} \simeq k_{t, i}^{2}>\tau$ is just a limit on the maximum emitted transverse momentum: this implies 'stopping' the DGLAP [11] parton evolution at a scale $\tau Q^{2}$, which gives single collinear logarithms.

These aspects are illustrated in the first order result for $\sigma(\tau)$ :

$$
\begin{aligned}
\frac{\sigma(\tau)}{\sigma}=1-\frac{\alpha_{\mathrm{S}} C_{F}}{2 \pi}\left[2 \ln ^{2} \frac{1}{\tau}-\right. & \left.3 \ln \frac{1}{\tau}\right] \\
& -\frac{\alpha_{\mathrm{S}}}{2 \pi} \frac{\ln 1 / \tau}{q(x)} \int_{x}^{1} \frac{d z}{z}\left[q\left(\frac{x}{z}\right) P_{q q}(z)+g\left(\frac{x}{z}\right) P_{q g}(z)\right] .
\end{aligned}
$$

The first line contains the soft and collinear double logarithms (which turn out to be identical to the $e^{+} e^{-}$result) from the conditions on the $\alpha_{i}$ and $\beta_{i}$. The second line contains the collinear logarithm associated with the restriction on the DGLAP evolution. Photon-gluon fusion plays a role only through this collinear logarithm, in the convolution with the gluon distribution.

The actual details of the resummation will be presented in [4]. Schematically, the result is

$$
\frac{\sigma(\tau)}{\sigma}=\left[1+\alpha_{\mathrm{S}} C(x)\right] \frac{q\left(x, \tau Q^{2}\right)}{q\left(x, Q^{2}\right)} \Sigma(\tau), \quad \Sigma(\tau)=e^{-\frac{\alpha_{\mathrm{S}} C_{F}}{2 \pi}\left[2 \ln ^{2} \frac{1}{\tau}-3 \ln \frac{1}{\tau}\right]+\mathcal{O}\left(\alpha_{S}^{2} \ln ^{3} \frac{1}{\tau}\right),}
$$

where $\Sigma(\tau)$ is just the corresponding $e^{+} e^{-}$quantity of [1] and $C(x)$ is a $\tau$-independent coefficient function which is given in 4 . The ratio $\frac{q\left(x, \tau \vec{Q}^{2}\right)}{q\left(x, Q^{2}\right)}$ comes from the suppression of radiation with $k_{t}^{2}>\tau Q^{2}$, as mentioned above.

\section{Comparison with fixed order programs.}

Expanding (6) to NLO it is possible to perform a comparison to the fixed order Monte Carlo programs DISASTER++ [8] and DISENT [9]. We actually look at the coefficient of $\left(\alpha_{\mathrm{S}} / 2 \pi\right)^{2}$ in

$$
\frac{\tau}{\sigma_{0}} \frac{d \sigma(\tau)}{d \tau}
$$

where we have normalised to the the Born cross section $\sigma_{0}$ for simplicity. At second order eq. (7) contains terms $\ln ^{n} \tau, n \leq 3$ and for the resummation to be correct to next-to-leading order we should correctly control terms $1 \leq n \leq 3$. So the difference between our expanded result and the exact result should at most be a constant for small $\tau$. The upper two plots of figure 1 show our results for the quark and gluoninitiated components of the answer compared to the predictions from DISASTER ++ . The shape of the distributions is well reproduced for small $\tau$. The lower two plots show the difference between the DISASTER ++ results and ours: one sees that for small $\tau$ 
this difference is indeed compatible with a constant, as required. Comparisons have also been made with DISENT, which seems to disagree with our result in the gluon sector at the level of a term proportional to $\ln ^{2} \tau$, and perhaps also in the quark sector.
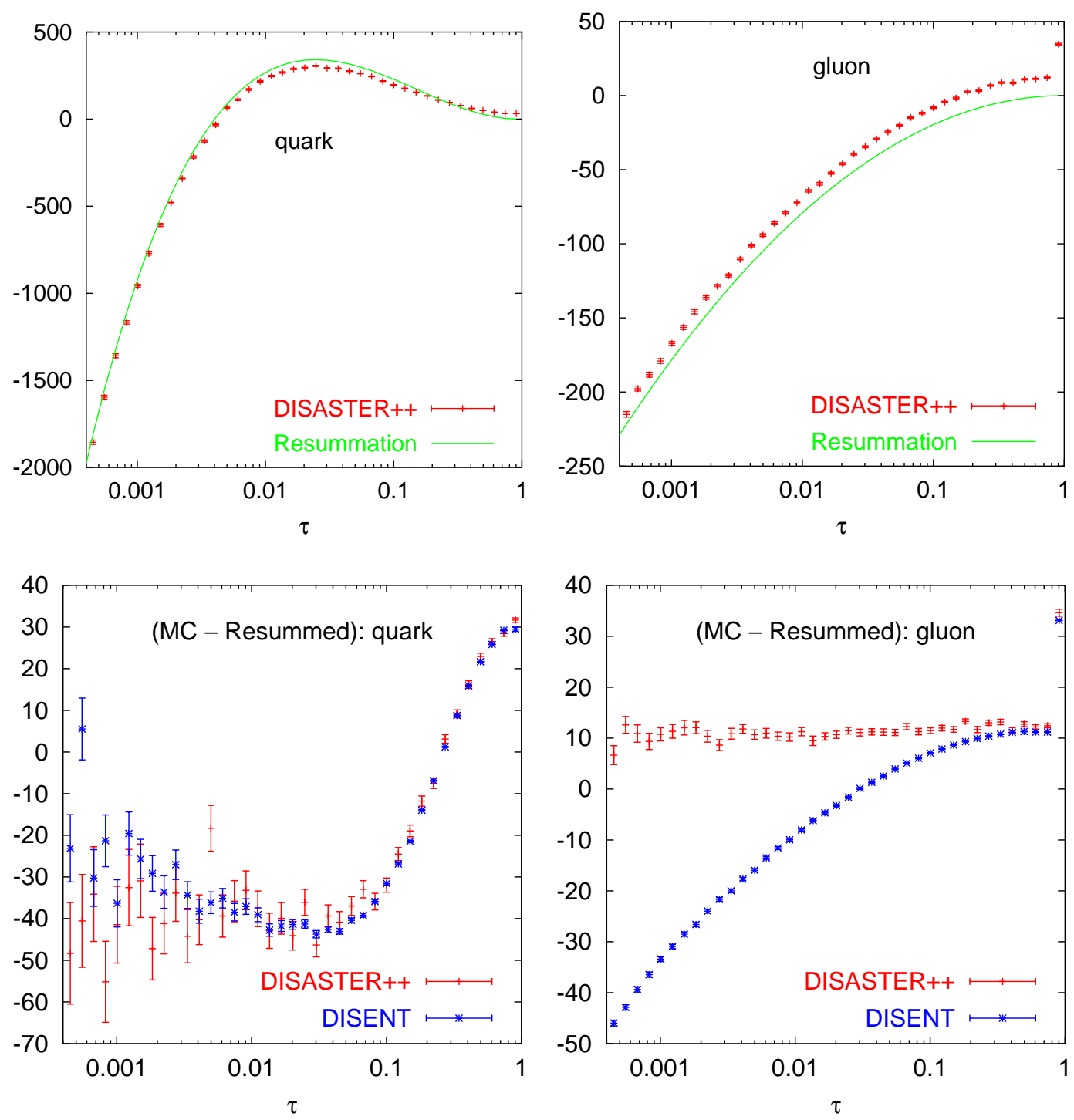

Figure 1. Coefficient of $\left(\frac{\alpha_{\mathrm{s}}}{2 \pi}\right)^{2}$ in $\frac{\tau}{\sigma_{0}} \frac{d \sigma}{d \tau}$. See text for details.

\section{Outlook}

Although we have results for the resummation of the thrust distribution in DIS, some work remains to be done for the practical implementation of our results for comparison with experimental data. In particular, prescriptions need to be defined for the matching of our resummed result with the fixed order results, in order extend the range of applicability of the calculation (without the matching the results are applicable only to very small $\tau$ ). 
Work is also in progress on the resummation of other DIS event-shape variables. Among these, the thrust normalised to the energy in the current hemisphere is close to completion. Other variables to be studied include the jet mass, the $C$-parameter and the jet-broadening (the resummation of the latter is relevant also for predicting the form of the power correction [12]).

Once this programme is complete, we hope that it will lead to phenomenological studies analogous to those already being carried out in $e^{+} e^{-}$.

\section{Acknowledgements}

We benefited much from continuous discussions of this and related subjects with Yuri Dokshitzer, Pino Marchesini and Mike Seymour. One of us (VA) is grateful to Yuri Dokshitzer and Pino Marchesini for introducing him to perturbative QCD, and to the organisers and the participants of the 1999 U.K. Phenomenology Workshop on Collider Physics for the invitation and for the stimulating atmosphere. He would also like to thank N. Brook and V. Khoze for useful discussions. This research was supported in part by MURST, Italy and by the EU Fourth Framework Programme 'Training and Mobility of Researchers', Network 'Quantum Chromodynamics and the Deep Structure of Elementary Particles', contract FMRX-CT98-0194 (DG 12-MIHT).

\section{References}

[1] S. Catani, L. Trentadue, G. Turnock and B.R. Webber, Nucl. Phys. B 407 (1993) 3.

[2] P.A. Movilla Fernandez, O. Biebel and S. Bethke, contributed to International Europhysics Conference on High-Energy Physics (EPS-HEP 99), Tampere, Finland, hep-ex/9906033.

[3] H1 collaboration (C. Adloff et al), Phys. Lett. B 406 (1997) 256, hep-ex/9706002; R.G. Waugh (on behalf of the ZEUS collaboration), in Brussels 1998, Deep inelastic scattering and QCD, p. 506.

[4] V. Antonelli, M. Dasgupta and G.P. Salam, Bicocca-FT-99-32, in preparation.

[5] Feynman R.P. 1972 Photon Hadron Interactions (W. A. Benjamin, New York); Dokshitzer Yu. L., Khoze V.A. and Troyan S.I. 1989,in Perturbative Quantum Chromodynamics (ed. A. H. Mueller, World Scientific, Singapore).

[6] S. Catani, Yu.L. Dokshitzer and B.R. Webber, Phys. Lett. B 322 (1994) 263.

[7] S. Catani, G. Marchesini and B.R. Webber, Nucl. Phys. B 349 (1991) 635.

[8] D. Graudenz, hep-ph/9710244.

[9] S. Catani and M.H. Seymour, Nucl. Phys. B 485 (1997) 291, [hep-ph/9605323].

[10] M. Dasgupta and B.R. Webber, JHEP 10 (1998) 001 [hep-ph/9809247.

[11] V.N. Gribov and L.N. Lipatov, Sov. J. Nucl. Phys. 15 (1972) 438; G. Altarelli and G. Parisi, Nucl. Phys. B 126 (1977) 298;

Yu.L. Dokshitzer, Sov. Phys. JETP 46 (1977) 641.

[12] Yu. L. Dokshitzer, G. Marchesini and G. P. Salam, Eur. Phys. J. direct C3 (1999) 1 hep$\mathrm{ph} / 9812487$. 